\title{
ANN Modelling on Vulnerabilities Detection in Code Smells-Associated Android Applications
}

\author{
Aakanshi Gupta $^{1}$, Deepanshu Sharma ${ }^{2}$, Kritika Phulli ${ }^{3}$
}

\begin{abstract}
There has been a lot of software design concerns in recent years that come under the code smell. Android Applications Developments experiences more security issues related to code smells that lead to vulnerabilities in software. This research focuses on the vulnerability detection in Android applications which consists of code smells. A multi-layer perceptron-based ANN model is generated for detection of software vulnerabilities and has a precision value of $74.7 \%$ and $79.6 \%$ accuracy with 2 hidden layers. The focus is laid on 1390 Android classes and involves association mining of the software vulnerabilities with android code smells using APRIORI algorithm. The generated ANN model The findings represent that Member Ignoring Method (MIM) code smell shows an association with Bean Member Serialization (BMS) vulnerability having 86\% confidence level and 0.48 support value. An algorithm has also been proposed that would help developers in detecting software vulnerability in the smelly source code of an android applications at early stages of development.
\end{abstract}

Keywords. Software Vulnerabilities, Code Smells, Android, ANN, Apriori Algorithm, Deep Learning, Machine Learning.

\section{Introduction and Motivation}

The popularity and vast usage of Android applications make it a platform of interest for the hackers to breach in and acquire unlawful gains. Thus, the vulnerabilities cannot be sidelined and are ought to be taken into consideration during the SDLC (Software Development Life Cycle) [22],[31]. It can be a design issue, and error-prone state, or any bad practice of code that can be easily tampered or mutated to perform breaches into the system. They can act as a manifest for malicious use of the application, which is not evident but require ingenuity to be exploited. Some studies [33], [40] state that $60 \%$ of breaches involve

${ }^{1}$ ASET, GGSIPU, New Delhi, India; aakankshi@gmail.com

2 Executive Branch-IT, Indian Navy, sharma.deepanshu97@gmail.com

${ }^{3}$ Application Development Associate, Accenture, India, kritika1298phulli@gmail.com 
vulnerabilities, with data breaches that cost enterprises an average of $\$ 3.92$ million. A typical user has a $27.9 \%$ chance of experiencing a data breach affecting a minimum of 10000 records [33]. With the exponential growth of vulnerabilities in 2019, which account for 17,220 in number which is $155 \%$ more as compared to 2016 [40], it is evident that security and quality of the software, both developed in an inclusive environment, requires to be elevated with better protocols. It is not just the software vulnerabilities that impinge an android application. However, there are several bad implementation choices and low levels of coding practices undertaken by a developer during the software product's implementation, affecting software quality and integrity. These practices lead to code smells - a term first coined by Kent Beck and Martin Fowler [9], [26]. Some researchers have extensively recognized code smells as a hindrance in software maintenance, which leads to lower productivity and higher re-work for developers. According to Yamashita et al. [44], around 32\% of the developers are unaware of code smells and their negative impacts on the software. The research studies available in public domain have focused on software vulnerabilities and Android code smells independently and haven't examined their relationship with each other or their cooccurrences in the software systems.

Focusing on the vulnerability issues and design flaws that exists in Android applications, this study aims to determine an associative relationship of the vulnerabilities in smelly codes using Apriori data mining technique. The relationship between code smells and vulnerabilities has been clearly defined as a positive correlation in the research [2]. In the field of software systems, vulnerability can be called a weakness or a state of exposure to threats such as hackers, viruses or unauthorized actions within a system whereas Code smells are another factor, that can adversely affect the maintenance phase of software system.

A robust machine learning model is essential to extract useful information about the occurrence of vulnerability in the smelly code. An Artificial Neural Network (ANN) is often advantageous over other approaches as it doesn't demand any specific assumptions about the error terms and the functional form (e.g., identically, and independently normally distributed) as compared to parametric approaches (discriminant analysis and regression equation) [18]. In contrast, a neural network model doesn't account for any assumption before modelling. Secondly, ANNs are approximators for universal functions to any desired level of accuracy. Neural networks are capable of capturing complex statistical interdependency among variables due to their capability of capturing non-linear relationships in data [19]. Considering the substantial growth in Android market and the effect of code smells and vulnerabilities on the integrity, security, and maintainability of the software, the focus of this study would be confined to Android-specific Java-based Applications as Java registers itself as the most preferred language for Android specific mobile application development [25].

Hence, this study targets at designing an ANN (Artificial Neural Network) backed with a multilayer perceptron network. This MLP- ANN modelling entitles to be the mainframe of the algorithm proposed for detecting the existence of software vulnerabilities in smelly code classes. Moreover, it inclines at corroborating to enhance the current knowledge about the phenomenon of co-occurrence of software vulnerabilities and Android specific code smell by employing the Association Mining for better in-sights of statistical dependencies to the developer. This advances the software quality and the overall functionality of android applications. The algorithm proposed in this research has the potential to be an integral part of the SDLC for expert code analysis and pre-empting any future attacks on the Android application systems. 


\section{Research Contributions:}

1. Generated Multilayer Perceptron Artificial Neural Network (MLP- ANN) model for detection of the occurrence of vulnerability in associated smelly code.

2. Calculated a precision value of $74.7 \%$ with $79.6 \%$ accuracy in the vulnerability detection using the developed ANN model.

3. Achieved the $86 \%$ confidence level and 0.48 support value for the association between 'Member Ignoring Method' (MIM) code smell and 'Bean Member Serialization' (BMS) vulnerability.

4. Proposed an algorithm for vulnerability detection of code-smell affected classes in android applications by employing the MLP- ANN model.

Structure of the paper: The paper is organized as follows: Section 2 describes the related work. Section 3 presents the methods and materials, Section 4 explains the experimental setup for this research. Section 5 presents the result and discussion of the study, Section 6 discusses the threats to validity, Section 7 presents conclusion and future scope.

\section{Related Work}

Software vulnerabilities and code smells always been an alarming aspect that has a very high potential to affect the software quality of the software. This has been a concerning facet, which has pushed researchers to analyse and extract better information for improved knowledge about the same. The researchers have contributed significantly by examining the vulnerabilities and suggesting the improvements in terms of vulnerabilities detection models [13], [20], [22], [24]. Recently many studies [22], [39] have been published focusing on the security vulnerabilities that affect the Android OS. Vulnerabilities detection tools are also available in the literature using different coding practices [22, 31]. Software vulnerabilities has also been detected using machine learning classification algorithms by Gupta et. al. [11]. A study by Minhaz and F. Zibran [16] presents a comparative analysis of different types of cloned and non- cloned codes-based vulnerabilities. Chowdhury et al. [5] aim to discover whether the three CCCs (Complexity, Coupling, Cohesion) metrics can be utilized as an early indicator of vulnerabilities within a software system.

The research work in Android software doesn't only sum up to vulnerabilities problems but also pose a threat in design flaw such as code smells. Several research studies [26], [31], [41]] advanced in the domain of code smells detection. Martin Fowler being the first to analyse these poor design implementations in software systems [9]. Following the path laid down by Martin Fowler, numerous researchers proposed different methods for analysing code smells in source code [11], [20], [41] The studies focusing on code smells have advanced from automated detection and refactoring to rule-based classification [20]. FA Fontana [7], compared the different machine learning approaches for the detection of code smells. F. Palomba [26] developed a tool is known as 'aDOCTOR', which helped in the detection of code smell specific to Android. Some studies [20], [43] have also established relationship between software vulnerabilities and code smells. Deep learning has proven to be an intrinsic part of artificial intelligence and has been acknowledged by many researchers [1], [19], [30] for better predictive analysis. Considering enhancement in software security 
and quality as a primary objective, many research studies [14], [30], [42] have employed Neural Network-based models for security assessment of software designs [1]. Not only this, but Researchers like Wang, Qi, and Seung Baek have also estimated the software quality and predicted the software effort in their studies with the help of the Neural Network model, proving it as a widely exercised machine learning technique for predictive analysis [30, [42]. In a study by A Adebiyi et. al. an attempt was made to assess the security design of the software using neural network.[1] A research conducted by Mehdi Khashei and Mehdi Bijari represents the time series forecasting model using neural network [18]. Table 1 summarizes the comparison based on various grounds between the proposed research and the previous researches.

Table 1: Comparasion between the proposed study and other studies

\begin{tabular}{|c|c|c|c|c|c|c|}
\hline & 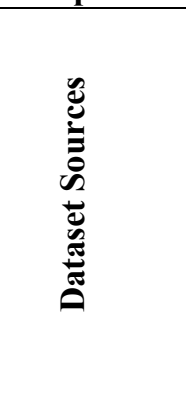 & 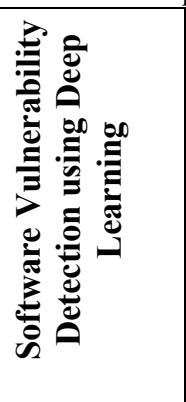 & 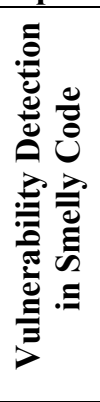 & 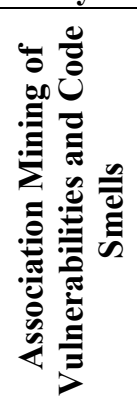 & 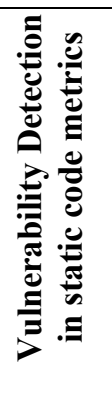 & 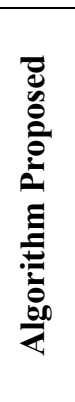 \\
\hline $\begin{array}{r}\text { Proposed } \\
\text { Approach } \\
\end{array}$ & Github & $\begin{array}{c}\text { Yes (Java- } \\
\text { Android) } \\
\end{array}$ & Yes & Yes & Yes & Yes \\
\hline $\begin{array}{l}\text { Li Z et al } \\
(2018)[20]\end{array}$ & NIST & $\begin{array}{c}\text { Yes } \\
(\mathrm{C} / \mathrm{C}++)\end{array}$ & No & No & No & No \\
\hline $\begin{array}{c}\text { Fang Wu. Et al } \\
(2017)[43]\end{array}$ & $\begin{array}{c}\text { Binary } \\
\text { Programs in } \\
\text { 32-bits } \\
\text { Linux } \\
\text { Machine }\end{array}$ & Yes (C) & No & No & No & No \\
\hline $\begin{array}{c}\text { Pang Y. et al. } \\
\text { (2017) [29] }\end{array}$ & F- DROID & $\begin{array}{c}\text { Yes (Multi- } \\
\text { platform } \\
\text { Programs) }\end{array}$ & No & No & No & No \\
\hline $\begin{array}{c}\text { Jacob A. } \\
\text { Harer et } \\
\text { al. }(2018) \\
{[12]}\end{array}$ & $\begin{array}{l}\text { Debain, } \\
\text { Github }\end{array}$ & $\begin{array}{c}\text { Yes } \\
(\mathrm{C} / \mathrm{C}++)\end{array}$ & No & No & No & No \\
\hline $\begin{array}{l}\text { Saccente } \\
\text { et. al [36] }\end{array}$ & $\begin{array}{c}\text { NIST } \\
\text { Juliet } \\
\text { Java test } \\
\text { suite }\end{array}$ & $\begin{array}{c}\text { Yes } \\
\text { (Java) }\end{array}$ & No & No & No & No \\
\hline
\end{tabular}




\section{Methods and Materials}

\subsection{Methodology}

This research intends to develop an algorithm for the detection of security vulnerabilities in the associated classes possessing code smells The Artifical Neural Network model supported by a multilayer perceptron (MLP- ANN) network acts as the main-frame of the algorithm facilitating software metrics as an essential part of the analysis. Consequently, this re-search emphasizes the co-occurrence of Android code smell with the highest modal score and software vulnerabilities. This helps to analyse the statistical dependency of both code smell and vulnerabilities on one another, which can be further exploited to gather more knowledge about their connection. Figure 1. Illustrates the workflow considered in due course of this study.

Initially, the applications were extracted from the open-source repository platform GITHUB and were analyzed to detect the presence of the code smells and vulnerabilities. Tools used to detect them are aDoctor and PMD.

aDoctor- https://github.com/fpalomba/aDoctor;

PMD-https://sourceforge.net/projects/pmd/.

The detection mechanism is performed with the help of code analyser tools. The detected Android code smells and software vulnerabilities were labelled alongside their corresponding classes and then further evaluated for the association rule between the code smell with the highest modal score and the considered software vulnerabilities.

Modal Score $=$ Highest number of classes containing a particular code smells

Association mining was performed to shape the relationship between smell and vulnerabilities. Further, the associated rules (vulnerability and code smell), along with their software metrics, were evaluated, and then, they were utilized to design the multilayer perceptron-based ANN model. The results of the entire study were exercised to devise an algorithm for the detection of vulnerabilities in the smelly code classes utilizing the information extracted formerly. 


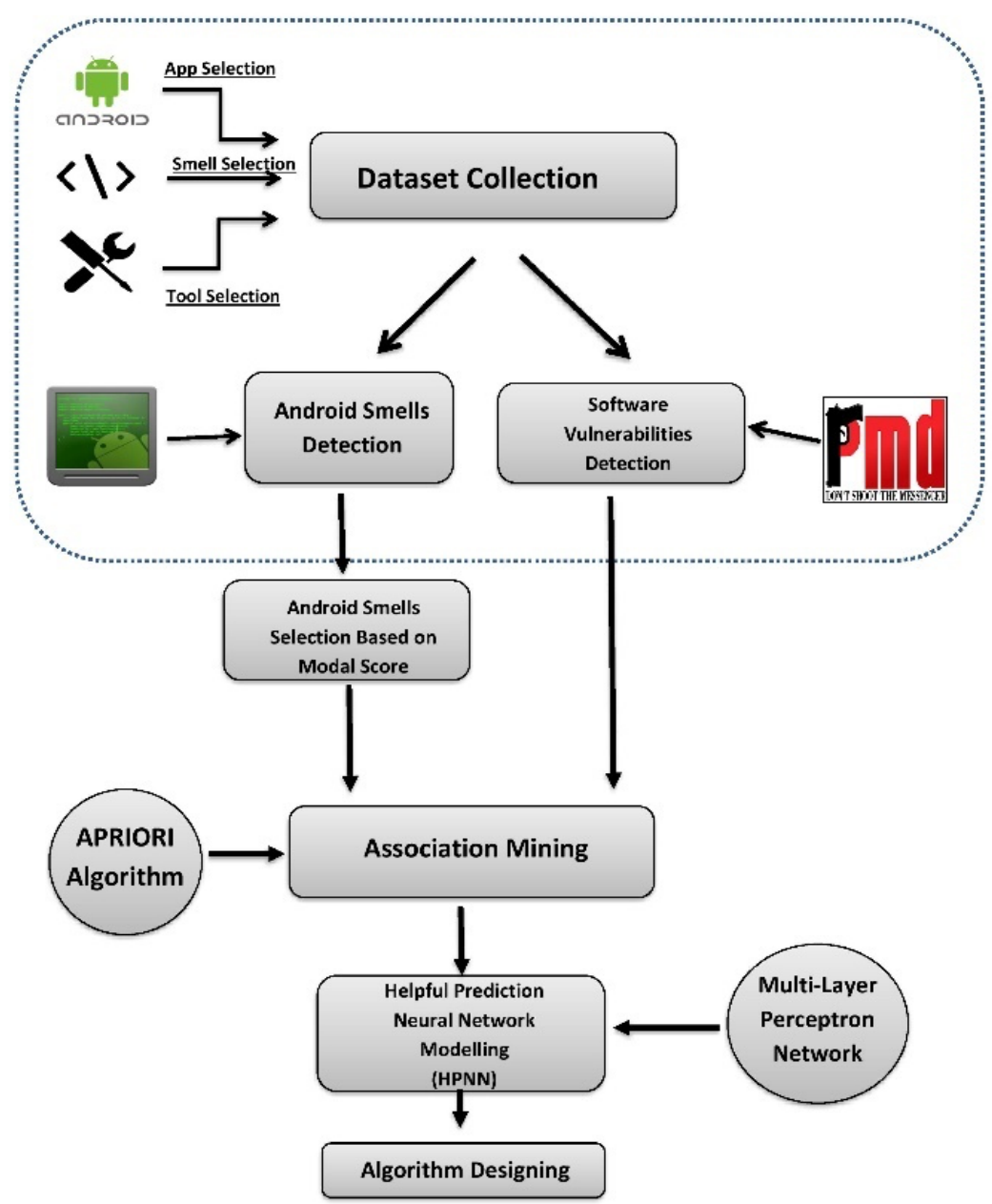

Figure 1. Workflow of the Study

\subsection{Context Selection}

The core of this study is focused on the Software vulnerabilities and the Android Specific Code Smell; in this context, this section is aimed at Application Selection, Software vulnerability Selection, and Android Code Smells Selection.

\subsubsection{Application Selection}

The study incorporates open-source Android software systems developed upon Java Programming Language and extracted from the open-source global repository, GitHub. 10Android applications were acquired from their respective GitHub repositories consisting of 1390 classes and 5500 methods. Table 2 consists of the selected applications with their statistical data. These open-source applications can be found on Google Play Store as well, 
with an average rating of 3.5 and above. To streamline the focus on the Android code that utilizes the basic functionalities that are commonly used in most of the applications and can be vulnerable to different defects, the general utility-based application categories were chosen for analysis like Audio Recorder App, Messaging App, Planner App, Social Media App, etc.

Table 2: Java Based Android Applications.

\begin{tabular}{|c|c|c|c|c|}
\hline Name of Application & Description & $\stackrel{0}{\stackrel{0}{*}}$ & $\begin{array}{l}\tilde{y} \\
\vdots \\
\vdots \\
\vdots \\
\vdots \\
\#\end{array}$ & 弟 \\
\hline AudioRecorder & $\begin{array}{l}\text { Open-Source Sound } \\
\text { Recorder Application }\end{array}$ & 71 & 254 & 1263 \\
\hline Cocoin App & $\begin{array}{l}\text { Muli-View Accounting } \\
\text { Application }\end{array}$ & 99 & 374 & 1176 \\
\hline ClipStack & $\begin{array}{l}\text { A Tiny Clipboard History } \\
\text { Manager Application }\end{array}$ & 23 & 93 & 304 \\
\hline LoginDemo & $\begin{array}{l}\text { Login System Module } \\
\text { Application }\end{array}$ & 7 & 10 & 27 \\
\hline MinimalTo-Do & $\begin{array}{l}\text { Open Source To-Do-List } \\
\text { Application }\end{array}$ & 24 & 43 & 162 \\
\hline ResturantBot & $\begin{array}{l}\text { Resturant Chat-Bot } \\
\text { Application }\end{array}$ & 28 & 53 & 183 \\
\hline SoundRecorder & $\begin{array}{c}\text { Sound Recorder } \\
\text { Application for Android }\end{array}$ & 13 & 38 & 105 \\
\hline Termux & $\begin{array}{l}\text { Android Terminal and } \\
\text { Linux Environment }\end{array}$ & 13 & 43 & 180 \\
\hline Timber & $\begin{array}{l}\text { Material Design Music } \\
\text { Player }\end{array}$ & 163 & 424 & 1725 \\
\hline Ucrop & $\begin{array}{l}\text { Image Cropping Library } \\
\text { for Android }\end{array}$ & 30 & 58 & 375 \\
\hline & & 471 & 1390 & 5500 \\
\hline
\end{tabular}

\subsubsection{Software Vulnerability Selection}

In this research, the focus is trimmed to the 3 different types of vulnerabilities which belong to the following domains: error-prone, design-flaw and bad-coding style [21]. The considered vulnerabilities are as follows: Bean Member Serialization (BMS), Law of Demeter (LD), and Local Variable Finalization (LVF) [11]. The vulnerabilities are described in Table 3 and had the highest frequency in the software system, implying higher proneness. They were examined at the class-level. The understanding of selected vulnerabilities relies on an automated static code analyser called PMD (Programming Mistake Detector) 
(https://sourceforge.net/projects/pmd/). A total of 884 considered vulnerabilities were detected in 10 Android-based Java applications.

Table 3: Software Vulnerabilities Description

\begin{tabular}{|c|l|c|}
\hline Abbreviation & Name & Description \\
\hline BMS & Bean Member Serialization & $\begin{array}{c}\text { If a class is a bean, it needs } \\
\text { to be serializable using } \\
\text { transient, static. }\end{array}$ \\
\hline LD & Law of Demeter & $\begin{array}{c}\text { The Law of Demeter is a } \\
\text { rule, that says "only talk to } \\
\text { friends". It helps to reduce } \\
\text { coupling between classes or } \\
\text { objects. }\end{array}$ \\
\hline LVF & Local Variable Finalization & $\begin{array}{c}\text { A local variable assigned } \\
\text { only once can be declared } \\
\text { final. }\end{array}$ \\
\hline
\end{tabular}

\subsubsection{Android Code Smells Selection}

In the research literature, the significant disruption on Android code smells was laid down by Reimann et al. [32]. This study takes 5 Android code smells into account [32]; namely, Member Ignoring Methods (MIM), Unclosed Closable (UC), Slow Loop (SL), No Low Memory Resolver (NLMR) and Leaking Inner Class (LIC) which according to their interpretation might get snubbed by a developer during rapid development. These smells have been selected based on their good modal scores and detected by aDoctor tool. Table 4 describes the considered code smells. The five code smells are being considered here MIM, UC, SL, NLMR and LIC.

Table 4: Android Code Smells Description

\begin{tabular}{|c|c|c|}
\hline Abbreviation & Code Smells & Description \\
\hline NLMR & $\begin{array}{l}\text { No Low Memory } \\
\text { Resolver }\end{array}$ & $\begin{array}{l}\text { Classes which do not possess } \\
\text { onLowMemory resolver for Low Memory } \\
\text { scenarios. }\end{array}$ \\
\hline SL & Slow Loop & Methods that use slow for loops. \\
\hline $\mathrm{UC}$ & Unclosed Closable & $\begin{array}{l}\text { Objects that tend to get closed and are } \\
\text { not closed after usage. }\end{array}$ \\
\hline MIM & $\begin{array}{l}\text { Member Ignoring } \\
\text { Methods }\end{array}$ & $\begin{array}{l}\text { Methods that ignore its members that } \\
\text { are defined and are not used. }\end{array}$ \\
\hline LIC & $\begin{array}{l}\text { Leaking Inner } \\
\quad \text { Class }\end{array}$ & $\begin{array}{l}\text { When inner class can access outer class } \\
\text { all the time and cause memory leak }\end{array}$ \\
\hline
\end{tabular}




\section{Experimental Setup}

The selection of code smells and vulnerabilities advanced by various compelling outcomes which were then examined to quantify the extracted information into better knowledge about vulnerabilities and code smells. The focus was laid on the extraction of code smells and vulnerabilities prevailing in source code.

The selection of code smells was made based on their modal score, and the considered 5 code smells reflect the highest modal scores amongst the 15 smells analysed by aDoctor tool. The percentage composition of these smells in the considered dataset are: NLMR is $23.6 \%$, $\mathrm{SL}$ is $18.4 \%$, UC is $8.9 \%$, MIM is $55.9 \%$ and LIC is $22.3 \%$. A total of $483 \mathrm{Java}$ based Android class files were analysed for the existence of code smells and vulnerabilities, out of which $55.9 \%$ were affected by Member Ignoring Method (MIM) smell achieving the highest modal score of 270 , hence, it was selected for association mining with the considered vulnerabilities. The diffusion of code smells along with their modal scores for each smell individually are listed in table 5. The vulnerabilities in the considered Android class files were identified and labelled following the analysis made using PMD. The diffusion of the considered software vulnerabilities in the 483 android class files are BMS vulnerability is $31 \%$, LD vulnerability is $34 \%$ and LVF vulnerability is $35 \%$. It is worth noting that out of the 3 considered software vulnerabilities, Local Variable Finalization (LVF) vulnerability was diffused in the maximum number of class files with 312 files being affected by it.

Once the dataset about the presence of the considered code smell with highest model score and vulnerabilities in the Android class files was prepared, the first step was to mine the association rule for detection of the co-occurrence of code smell and vulnerabilities to outline the statistical relationship highlighting their association. The relationship established would further assist in modelling the multilayer perceptron network to detect software vulnerabilities in the associated smelly code.

Table 5: Android Code Smells Modal Scores

\begin{tabular}{|c|c|c|}
\hline Android Code Smells & $\begin{array}{c}\text { Diffusion of Code Smells } \\
(\mathbf{\%})\end{array}$ & Modal Score \\
\hline No Low Memory Resolver & 23.6 & 114 \\
\hline Slow Loop & 18.4 & 89 \\
\hline Unclosed Closable & 8.9 & 43 \\
\hline Member Ignoring Method & 55.9 & 270 \\
\hline Leaking Inner Class & 22.3 & 108 \\
\hline
\end{tabular}

\subsection{Associative Mining}

Association rule discovery belongs to the unsupervised learning technique for detecting local patterns that highlight conditions of attribute value occurring in a given dataset.[42], [44]. 
Formally,

let $A=\left\{a_{1}, a_{2}, a_{3}, \ldots \ldots, a_{n}\right\}$

$B=\left\{b_{1}, b_{2}, b_{3}, \ldots . . b_{n}\right\}$;

a set of $n$ binary attributes (items) indicating the presence of a specific property in the data under consideration,

let $C=\left\{c_{1}, c_{2}, c_{3}, \ldots . ., c_{m}\right\}$;

a set of $\mathrm{m}$ transactions indicating the analyzed element set.

This implies the association rule to be of the form

$$
X \rightarrow Y
$$

where $X \subseteq A, Y \subseteq B$, and $X \cap Y=\emptyset$.

In this research study, the set $\mathrm{C}$ is composed of all the class files considered for analysis. In contrast, each item in the set A indicates the presence of code smell, and B indicates the presence of vulnerabilities in transactions considered in the set $\mathrm{C}$.

This study deals with class level instances of the source code for analysis of association rule. It is to be noted that if the code smell and vulnerability affect the same class, they are said to be co-occurring.

In this work, the associative algorithm employed for mining is the Predictive apriori algorithm [38]. The concept of apriori property says that all subsets of a frequent itemset must also be frequent. The overall output of the associative algorithm is a set of rules explaining the co-occurrence of two items with a specific confidence value. In this study, the confidence value is considered as accuracy. The strength of the rule is also reflected by its support value [38]. Both confidence and support values help in quantitatively measuring the quality of the rule, which were calculated as per the given formula.

$$
\begin{gathered}
\text { Confidence }(X \rightarrow Y)=\frac{\operatorname{Support}(X \cup Y)}{\operatorname{Support}(X)} \\
\operatorname{Support}(X)=\frac{|\{t \in T ; X \subseteq t\}|}{|T|}
\end{gathered}
$$

Where $\mathrm{T}=$ total number of classes in the system

A data mining software named WEKA (Waikato Environment for Knowledge Analysis) was considered for the application of the associated data mining technique. WEKA consists of a collection of machine learning algorithms for data mining. The minimum metric score of 0.8 for confidence value was considered for this study, with an analysis of different confidence and support values. Table 6 describes the support and confidence values of association rule consisting of MIM code smell and 3 considered vulnerabilities as described in table 3. The best association rule extracted out of the 3 combinations of MIM Code Smell with Bean Member Serialization (BMS), Law of Demeter (LD), Local Variable Finalization (LVF) software vulnerabilities was further explored to consider their strong relationship into the account for developing the neural network model for detection of software vulnerability 
in associated smelly code. The MLP- ANN modeling unfolds the deep relationship of associated android code smell with software vulnerability.

Table 6: Support \& Confidence of MIM Smell Association with Software Vulnerabilities

\begin{tabular}{|c|c|c|c|}
\hline $\begin{array}{c}\text { Condition } \\
\text { Code Smell) }\end{array}$ & $\begin{array}{c}\text { Consequence } \\
\text { (Software Vulnerability) }\end{array}$ & Confidence & Support \\
\hline & Bean Member Serialization & 0.86 & 0.48 \\
\cline { 2 - 4 } $\begin{array}{c}\text { Member } \\
\text { Ignoring } \\
\text { Method }\end{array}$ & Law of Demeter & $\mathbf{0 . 8 0}$ & $\mathbf{0 . 4 5 1}$ \\
\cline { 2 - 4 } & Local Variable Finalization & $\mathbf{0 . 8 4}$ & $\mathbf{0 . 4 6 9}$ \\
\hline
\end{tabular}

\subsection{Pre-Modeling Phase}

Considering the above-stated scenario, the vulnerability detection modeling proctored by an MLP network was further executed on the classes of the best associated smelly code. A total of 1161 class level instances among 1390 were considered, incorporating the best associated smell. For modeling, the vulnerability detection network (ANN model) in associated smelly code, the software metrics of the smelly code were taken into consideration. The software metrics are crucial for measuring the features of software products and processes along with the flexibility, accuracy, complexity, extensibility. They have been essential for computing the quality of Java-based android applications [15, 37]. The software source code metrics were extracted at the class-level of considered applications, from a static code analyzer tool, SciTools Understand (https://scitools.com/). The generation of static metrics acts as a source of authentication for predicting and anticipating the enhancement of source code.

The dataset comprising of software metrics of the best-associated android code smell was prepared and mapped along with the presence of the best-associated software vulnerability with that smell. Out of the 3 considered software vulnerabilities, Bean Member Serialization (BMS) vulnerability marked the best association rule with the Member Ignoring Member (MIM) smell as shown in table 6.

Thus, the network was designed accounting the software metric of MIM affected classes intending to model the presence of $B M S$ vulnerability considering classes with smelly code.

\subsection{Neural Network Modeling}

Neural Networks (NN) is a deep learning-based artificial neural network-building algorithm that stimulates the human brain function. The network constitutes an input layer, an output layer, and the hidden layer between the input and output layers to facilitate the sophisticated features. It can deal with labeled and unstructured data in a seamless manner exhibiting the artificial learning backed mathematical functions. There are various deep learning 
architectures, including Multi-Layered Perceptron based Network (MLP), Deep Neural Network (DNN), Deep Belief Networks (DBN), Radial Basis Function Neural Network (RFN), Convolution Neural network (CNN). This study uses a Multi-Layered Perceptron (MLP) based Neural Network modeling technique for detecting software vulnerabilities.

\subsubsection{Multilayer Perceptron Network (MLP)}

The use of neural networks and the multilayer perceptron have shown to be an effective alternative to more traditional statistical techniques [30]. The multi-layer perceptron can be trained to approximately any measurable function. The exciting feature of the multilayer perceptron technique is that it makes noformer assumptions regarding the distribution of data. It can model highly non-linear functions and can be accurately trained to generalize the results when presented with unseen, new data [14]. These mentioned features of the MLP makes it highly suitable for developing predictive models. These mentioned features of the MLP makes it highly suitable for developing predictive models.

The multilayer perceptron constitutes a system of interconnected neurons, in a highly sophisticated net- work model that outlines the non-linear mapping be- tween the input and output attributes or parameters. The nodes exhibit connection with other nodes by synaptic weights governing their connection to pro- duce a discrete outcome. Between the input feeding, the nodes, and the output layer lie a virtual "gate" of simple non-linear function that controls the prediction of the outcome, this is termed as an activation function. It is a blend of many simple transfer functions that exhibit non-linear nature, enabling the neuron to estimate the extreme non-linear function. The output of a node is elevated by its synaptic weight and for- warded to be an input for another node in the next layer of the network. This highlights the direction of propagation of information as forward in nature, thus termed as feed-forward neural network; thus, this study makes use of feed-forward multilayer perceptron modeling technique [14]. Input is a feature vector ' $\mathrm{x}$ ' multiplied by weights ' $\mathrm{w}$ ' and summed to a bias

'b': $\mathrm{y}=\mathrm{w} \times \mathrm{x}+\mathrm{b}$.

A multilayer perceptron may consist of one or more hidden layers and an output layer with a single input layer virtually playing the role of a feeder of independent datasets using which the output is predicted. The below-mentioned equation depicts the output estimation of the multilayer perceptron model.

$$
y=\varphi\left(\sum_{i=1}^{n} w_{i} x_{i}+b\right)=\varphi\left(w^{T} x+b\right)
$$

Where,

$\mathrm{w}=$ weight vector,

$\mathrm{x}=$ input vector,

$\mathrm{b}=$ bias and

phi $=$ a non-linear activation function . 
The selection of a suitable set of transfer functions and demands specific assumptions about the error terms and the functional form and (e.g., identically and in- dependently normally distributed), plays a critical role in producing a smooth and measurable function between the input and output layers. The accuracy and complexity of the model highly depend on its activation functions and the number of hidden layers considered for the model. The Multilayer perceptron model facilitates learning through training and thus becomes an integral part of the supervised learning algorithms group. The dataset required for the same consists of inputs and their respective outputs to determine a pat- tern between them and draw out a model replicating the neural system of the human brain. The accuracy of the model is evaluated based on the number of correct predictions made by the model concerning the total number of input feeds. The prepared dataset that consists of the software metrics of the $M I M$ affected smelly code, and the respective $B M S$ vulnerability label alongside those classes is utilized in an attempt to prepare a vulnerability detection model using the mentioned software metrics as the attributes of the input layer. The 6-software metrics that have a critical significance in software academic literature were taken into consideration for the modelling of vulnerability in this study, namely: - SumCyclomaticModified, PercentLackOfCohesionModified, MaxInheritanceTree, CountDeclClassMethod, CountDeclClass Variable, CountLineCodeExe.

The dataset comprising of 1161 class level instances was divided into 7:3 for training and testing, respectively. The training type employed in this modeling is Batch type. The batch type training set updates the synaptic weights after passing all training data records; that is, batch training utilizes information from all records in the training dataset. Batch training is often preferred as it minimizes the total error. The testing plays a vital role in determining the accuracy of the model. Figure 2 depicts the neural network model designed to predict the presence of Bean Member vulnerability (BMS) in the associated MIM affected smelly code classes.

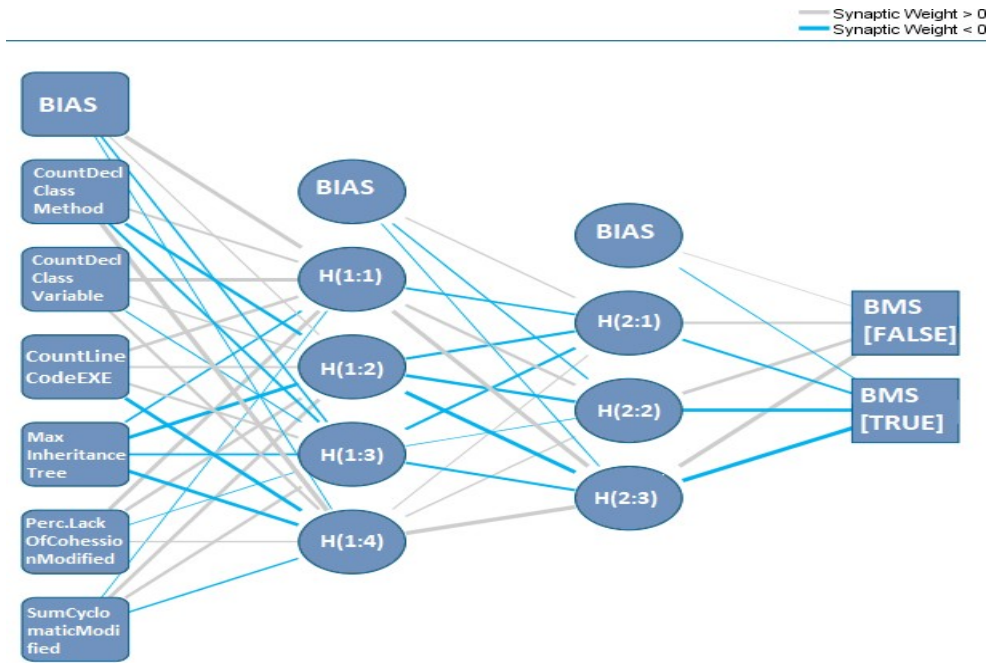

Figure 2. Neural Network layout of MLP Network 
The proposed network model consists of 2 Hidden Layers between the input and the output layer with 4 internal units of hidden layer 1 and 3 internal units of hidden layer 2 respectively. Moreover, the activation or the transfer function used in the hidden layer was Hyperbolic tangent function and the output layer was sigmoid function. A sigmoid function is a differentiable, bounded real function that has no negative derivative at each point and is defined for all real input values. A sigmoid "function" or a sigmoid "curve" refer to the same object. It is monotonic and has the first derivative of a bell shape. The equation 4 reflects a sigmoid formula.

$$
S(x)=\frac{1}{1+e^{-x}}=\frac{e^{x}}{e^{x}+1}
$$

It is to note that the training of the model was stopped when a situation of consecutive steps arrived with no decrease in error.The model was examined to evaluate 6 performance measures stated below:

- True Positive Rate (TP Rate): - It measures the correctly predicted positive values that are positive in nature.

- False Positive Rate (FP Rate): It measures the predicted positive values that are negative in nature.

- Recall: It is the fraction of the total amount of relevant instances that are retrieved.

- F-measure: It measures the weighted harmonic mean of precision and recall.

- Accuracy: It is the percentage of correctly predicted instances by the model.

Table 7 depicts the quantitative performance score of the generated MLP- ANN model. Table 8 shows the in-depth statistical results of the parameter estimates at each layer of the model. Figure 3 highlights the ROC curve chart which measures the performance of the neural network. ROC stands for "Recevier Operating Characteristics" which shows the importance of the test. The large area in the ROC curve also states the usefulness of the test..

Table 7: Performance measures of MLP Network Model

\begin{tabular}{|l|l|l|l|l|l|l|}
\hline Model & $\begin{array}{l}\text { TP- } \\
\text { Rate }\end{array}$ & $\begin{array}{l}\text { FP- } \\
\text { Rate }\end{array}$ & Precision & Recall & $\begin{array}{l}\text { F- } \\
\text { measure }\end{array}$ & Accuracy \\
\hline Training & 0.884 & 0.331 & 0.732 & 0.884 & 0.8 & 0.778 \\
\hline Testing & 0.884 & 0.248 & 0.763 & 0.884 & 0.83 & 0.815 \\
\hline & 0.884 & 0.289 & 0.747 & 0.884 & 0.81 & 0.796 \\
\hline
\end{tabular}




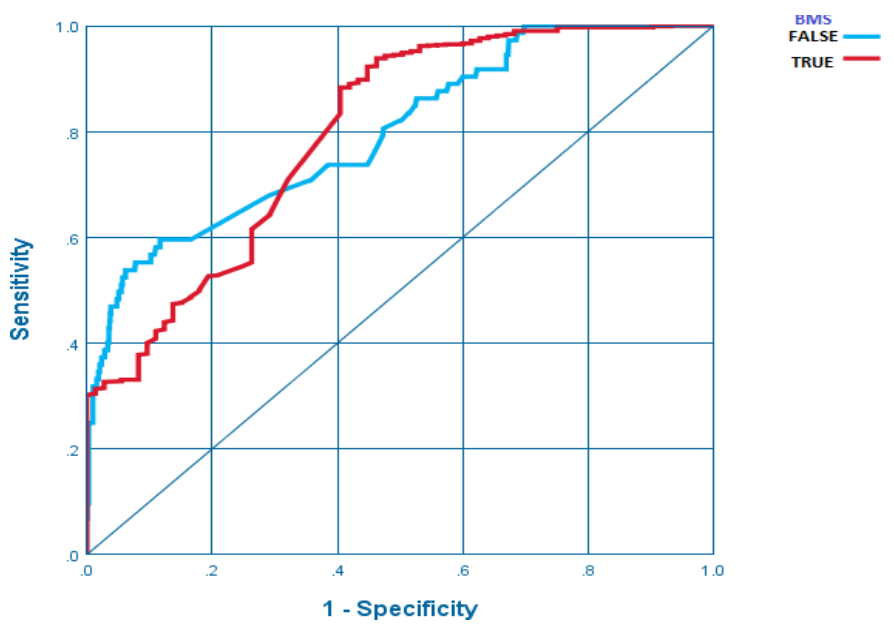

Figure 3. ROC Curve Chart 


\begin{tabular}{|c|c|c|c|c|c|c|c|c|c|c|c|c|c|c|c|c|}
\hline & & & 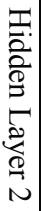 & & & & & 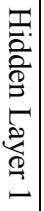 & & & & & & 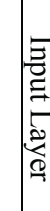 & & \\
\hline 吾 & 吾 & 蒙 & 冕. & 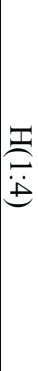 & $\mid$ & 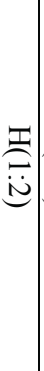 & 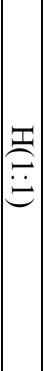 & $\begin{array}{l}\underset{\overrightarrow{\nexists ्}}{\tilde{E}} \\
\text {. }\end{array}$ & 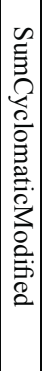 & 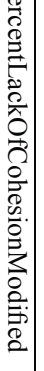 & 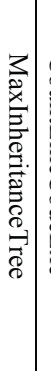 & 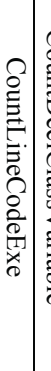 & 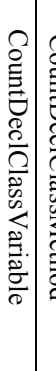 & 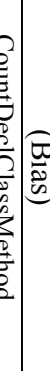 & & \\
\hline & & & & & & & & & $\begin{array}{l}b \\
\dot{\omega} \\
\perp \\
\perp\end{array}$ & 芯 & $\begin{array}{l}b \\
\vdots \\
\dot{t} \\
\dot{d} \\
0\end{array}$ & $\begin{array}{c}\overline{\mathrm{i}} \\
\vec{u}\end{array}$ & 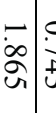 & $\vec{w}$ & 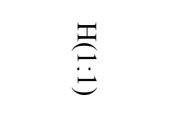 & \multirow{4}{*}{ 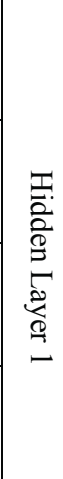 } \\
\hline & & & & & & & & & $\begin{array}{l}\overrightarrow{\vec{\Delta}} \\
\dot{\vec{\omega}} \\
\dot{u}\end{array}$ & $\dot{\bar{\sigma}}$ & $\begin{array}{l}\dot{\vec{u}} \\
\dot{\vec{v}}\end{array}$ & 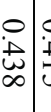 & 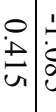 & bi & 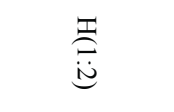 & \\
\hline & & & & & & & & & 点 & 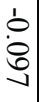 & 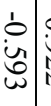 & 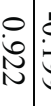 & 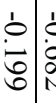 & $\overbrace{0}^{b}$ & 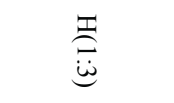 & \\
\hline & & & & & & & & & $\begin{array}{l}\dot{b} \\
\dot{w} \\
\hat{\sigma}\end{array}$ & 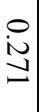 & $\begin{array}{l}\frac{1}{\tilde{n}} \\
\dot{u}\end{array}$ & $\begin{array}{l}\dot{u} \\
\dot{u} \\
=\end{array}$ & 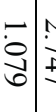 & $\frac{c}{d}$ & 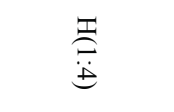 & \\
\hline & & & & i & $\begin{array}{l}1 \\
0 \\
0 \\
N \\
\text { N }\end{array}$ & $\begin{array}{l}1 \\
\dot{0} \\
\dot{0} \\
\end{array}$ & $\mid \begin{array}{l}0 \\
\stackrel{1}{+} \\
\dot{\omega} \\
0\end{array}$ & $\begin{array}{l}\stackrel{0}{w} \\
\pm \\
\pm\end{array}$ & & & & & & & 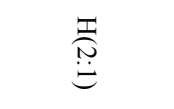 & \multirow{3}{*}{ 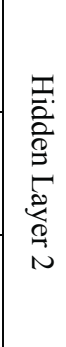 } \\
\hline & & & & i & $\begin{array}{l}0 \\
\dot{0} \\
\dot{2} \\
\omega \\
\omega\end{array}$ & 它 & $\mid \begin{array}{l}\overrightarrow{\dot{ }} \\
\infty \\
+\end{array}$ & $\begin{array}{l}\dot{b} \\
\dot{\omega} \\
\dot{D} \\
\omega\end{array}$ & & & & & & & $\underbrace{}_{\substack{\mathbb{N} \\
\stackrel{N}{*}}}$ & \\
\hline & & & & N & 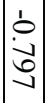 & $\begin{array}{l}\dot{\omega} \\
\dot{\vec{\alpha}}\end{array}$ & 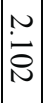 & $\begin{array}{l}\dot{b} \\
\dot{0}\end{array}$ & & & & & & & 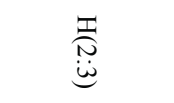 & \\
\hline $\mid \begin{array}{l}N \\
\tilde{N} \\
+\end{array}$ & $\begin{array}{l}\overrightarrow{\tilde{N}} \\
\tilde{\infty} \\
\infty\end{array}$ & $\begin{array}{l}0 \\
\infty \\
+ \\
\omega\end{array}$ & & & & & & & & & & & & & $\begin{array}{c}{[\text { Bean }} \\
\text { Member } \\
\text { Serializatio } \\
\mathrm{n}=\text { FALSE] }\end{array}$ & 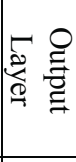 \\
\hline \begin{tabular}{|c|}
$\grave{1}$ \\
$\stackrel{0}{0}$ \\
$\stackrel{0}{0}$
\end{tabular} & $\mid \begin{array}{c}\dot{\dot{u r}} \\
\vec{\perp}\end{array}$ & & $\begin{array}{l}\dot{1} \\
\dot{\bar{U}} \\
\infty\end{array}$ & & & & & & & & & & & & $\begin{array}{c}{[\text { Bean }} \\
\text { Member } \\
\text { Serializatio } \\
\mathrm{n}=\text { TRUE] }\end{array}$ & \\
\hline
\end{tabular}




\section{Results and Discussion}

The study targets to discover the co-occurrence of software vulnerabilities and android specific code smells diffused in android application. A total of 1390 android classes were considered for the analysis which were extracted from open-source repository- Github. The diffusion of 3 software vulnerabilities were studied for android applications infected with 5 android specific code smells. Subsequently, this research has been broadly discussed in the following manner:

1. Association Mining: The association of the code smells with software vulnerabilities has been analyzed and investigated for the best association rule extract information about the relationship of the code smells with software vulnerabilities. This association mining was achieved by employing apriori algorithm from Weka data mining tool. It has been observed that around 483 class files were evaluated for the presence of the code smells and vulnerability. Out of these classes, $67 \%$ classes were affected with 5 android code smells and $76.3 \%$ were affected with the 3 software vulnerabilities. It is worth noting that out of the 5 android code smells, MIM smell resulted in highest modal score (270), which further helped in exploring the association with 3 considered software vulnerabilities. Out of the 3 possible combinations for assocition, the code smell ' $M I M$ ' and the software vulnerability ' $B M S$ ' exhibited best association rule with a confidence value of 0.86 and support value of 0.48 . This helps in inferring that $M I M$ code smell will co-occur with $B M S$ software vulnerability with an accuracy of $86 \%$.

$$
M I M=\text { True } \rightarrow \text { BMS }=\text { True }
$$

The support and confidence of other evaluated association rules have been listed in table 6. The study did not limit itself to the analysis of the association of code smell with vulnerabilities but also aimed to analyze the co-occurrence of the considered vulnerabilities within themselves.

$$
B M S=\text { True }+L D=\text { True } \rightarrow L M F=\text { True }
$$

The association of the software vulnerabilities with each other displayed a confidence value of 0.92 . This infers that if the $B M S$ vulnerability co-exists with the $L D$ vulnerability, there is a $92 \%$ possibility that the $L M F$ vulnerability will also exist.

Consolidating eq. (5) and eq. (6), it infers that the LMF vulnerability has an indirect association with MIM code smell. i.e., If the BMS vulnerability occurs with LD vulnerability for the class affected by MIM code smell (MIM code smells co-occur with BMS and LD vulnerability), then it is high probability that the LMF vulnerability will also co-occur. This relationship is depicted in Figure 4. The above inference validates that the association of MIM code smell with LMF vulnerability exists with 0.84 confidence value and 0.469 support value. 


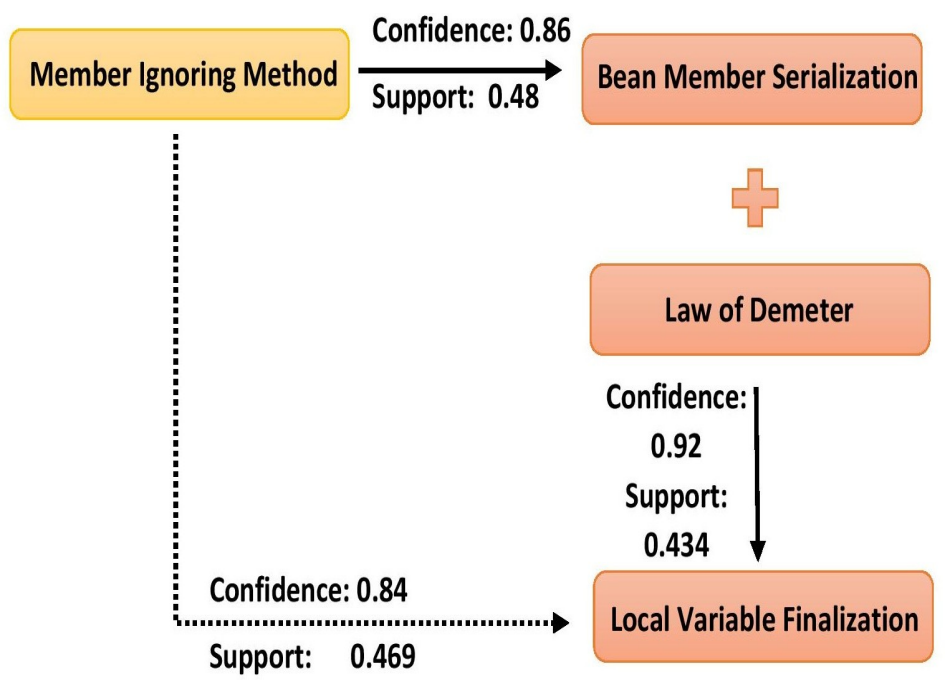

Figure 4. Association Flow Diagram of Code Smell with Software Vulnerabilities

2. Artificial Neural Network (ANN): Once, the association of code smells with software vulnerability has been discovered, the best-associated rule was extracted for further analysis. As mentioned in section 4, a multilayer perceptron Artifical Neural Network (MLP- ANN) model was designed. The parameters considered are as follows:

- Two (2) hidden layers employing sigmoid function

- Hyperbolic tangent function

- Input layer with 6 prominent software metrics of MIM code smells affected classes.

The network is shown in figure 2. The model portrays an accuracy of $79.6 \%$ and a precision of $74.7 \%$ which is significant to highlight the relationship between android specific code smells and software vulnerabilities. The MLP model obtained depicts the significance of independent variable by marking their normalized importance value. Table 9 depicts the importance of the independent variables used to detect $B M S$ software vulnerability in the MIM code smell. It highlights that CountDeclClassMethod metric holds maximum normalized importance with a value of 0.184 . Subsequently, figure 3 portrays the ROC curve graph of the MLP- ANN Model with Area Under ROC accounting to 0.857.

3. Algorithm Designing: The overall analysis performed for this study was then strategically devised to contrive an algorithm that makes use of the MLP- ANN modelling and the association mining of $M I M$ code smell with $B M S$ software vulnerability for detecting a vulnerable class which is affected by code smells. 


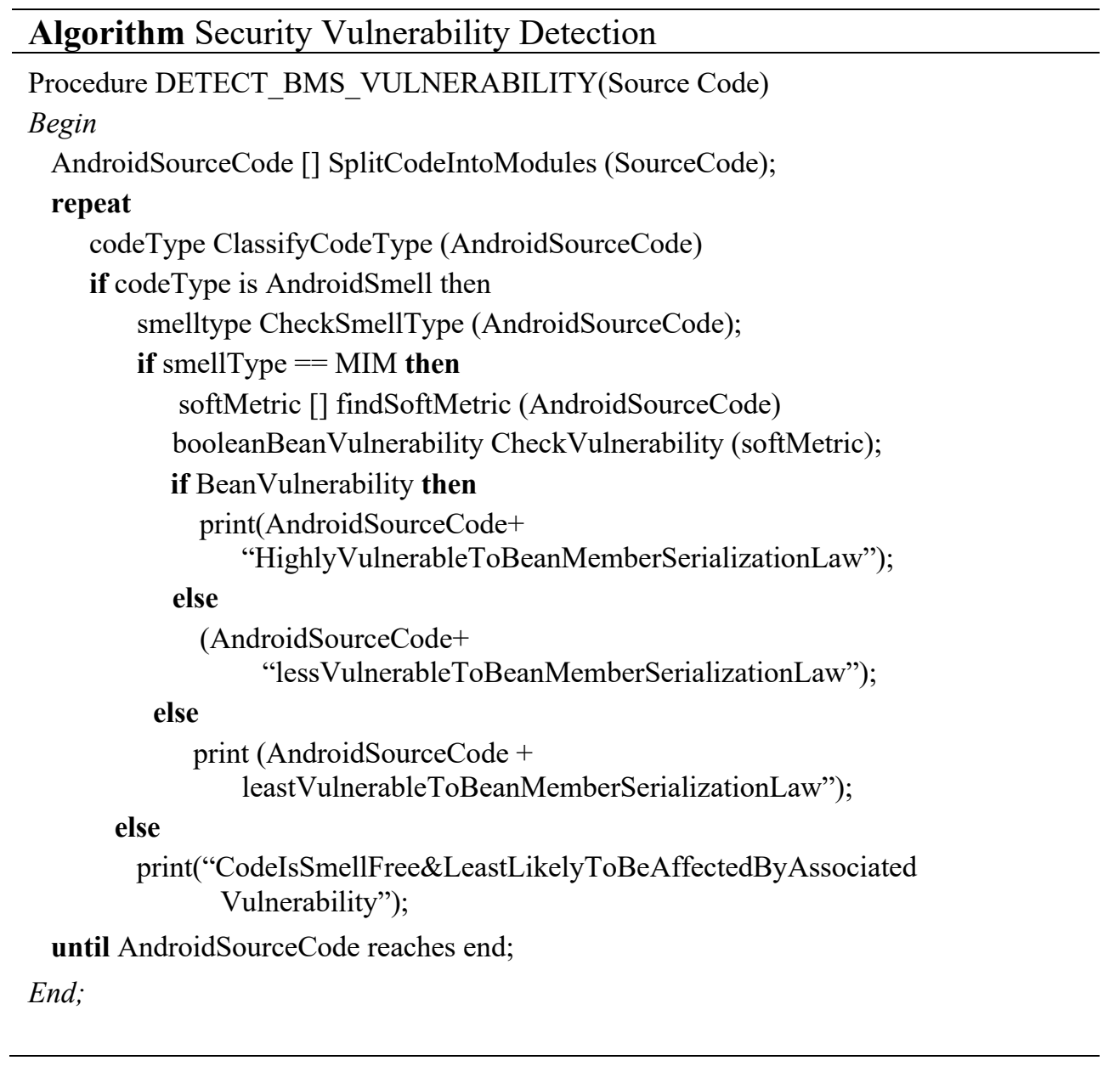

The above stated algorithm uses the associated rules and the designed MLP- ANN model to detect the presence of the vulnerability. It takes the source code as an input and breaks it into smaller modules for evaluation. It first analyzes for the presence of the smell, and if the smell exists in the code module, it inspects the associated smell. If the results are affirmative, the considered software metrics are calculated for the corresponding code module and fed to a function employing the Neural Network Model in its support. The Boolean value result returned by the function helps in detecting the presence of vulnerability in the smelly code.

\section{Threats to Validity}

This section undertakes the threats to the validity of the performed study which may disturb the validity of the proposed research work. This study outlines the relationship between android specific code smell and software vulnerabilities. The source could be the experimentation which certainly induces errors in the formulation of the dataset.The 
detection of code smells, and vulnerabilities are undertaken with the help of automated tools like aDoctor and PMD which incorrectly classify some classes, thus hampering the association but, the inaccuracy is well within a range of 5\% which would not significantly impact the model but can still be considered as a threat to this study. In addition the selection of metrics also depends upon the considered smells and vulnerabilities which can be varies accordingly if we consider the another smells and vulnerabilities.

However the size of the dataset which contains the smells could also act as a barrier in training the model. The number of hidden layers in the model can also challenge the results. Considering other code smells associated with these might alter the results obtained from this study. The study is based on classes of the android applications, thus limiting itself to the android platform.

\section{Conclusion and Future Scope}

This research study aimed to examine the associative relationship between the androidspecific code smell and the software vulnerabilities to draw out the statistical dependencies of both the factors. It is achieved by creating a multilayer perceptron network for detecting the presence of software vulnerabilities in the associated smelly code that affects the software security. The 5 code smells were initially taken into consideration for the evaluation, namely: Unclosed Closable, No Low Memory Resolver, Slow Loop, Leaking Inner Class, Member Ignoring Method, and 3 software vulnerabilities were taken namely Bean Member Serialization, Law of Demeter, and Local VariableFinalization. About 483 android class files were analyzed for the presence of the code smells mentioned above and vulnerabilities.

Table 9: Importance Score of Software Metrics in MLP Network

\begin{tabular}{|c|c|c|}
\hline SoftwareMetrics & $\begin{array}{c}\text { Importance } \\
\text { Score }\end{array}$ & Normalized Importance \\
\hline CountDeclClassMethod & 0.178 & $96.6 \%$ \\
\hline CountDeclClassVariable & 0.184 & $100.0 \%$ \\
\hline CountLineCodeExe & 0.145 & $78.9 \%$ \\
\hline MaxInheritanceTree & 0.170 & $92.6 \%$ \\
\hline PercentLackOfCohesionModified & 0.165 & $89.8 \%$ \\
\hline SumCyclomaticModified & 0.158 & $86.1 \%$ \\
\hline
\end{tabular}

Further, the association mining was employed between the smell with the highest modal score and 3 considered vulnerabilities, and the best association rule between the code smell with the highest modal score, and the vulnerabilities was fetched for further exploration. The focus was not just on the as- sociation of the code smell with vulnerabilities but also on the inter-association of the software vulnerabilities. MIM smell showed best association rule with Bean Member Serialization vulnerability with the confidence value of $86 \%$, and this association rule was further explored by designing a multilayer perceptron network of software vulnerabilities with software metrics of the smelly code. The model drawn with the 
help of the multilayer perceptron network had $79.6 \%$ accuracy and $74.7 \%$ precision with 2 hidden layers having the sigmoid function as its activation function.

The information, thus gathered from the above study was coined to propose an algorithm that would potentially find its application into the SDLC (Software Development LifeCycle) to better evaluate the code and detection of the software vulnerabilities in the smelly codes. The algorithm takes source code as the input file. It makes use of the association rule along with the designed neural network model to label out the presence of the vulnerability.

This algorithm, thus, can help the developers to flag out the presence of vulnerabilities in the source code. It can help them to eradicate these smells and vulnerabilities in the maintenance phase of the software, which would result in a secure and reliable application that would be less prone to the attacks. Further, this re- search can act as a motivation for future studies focusing on software vulnerabilities. It can lay down a path for different $\mathrm{ML}$ models for the detection of vulnerabilities in source code. It can also help the researchers explore the dependency of code smells with vulnerabilities, which can help them lay a foundation for an era of supreme quality software resilient to exploitation. This study can also be performed on different languages for the same to draw out the dependencies of the design flaws of language with vulnerabilities.

\section{References}

[1] Adebiyi A, Arreymbi J, Imafidon C. Security Assessment of Software Design using Neural Network. arXiv preprint arXiv:1303.2017, 2013.

[2] Aakanshi Gupta, Bharti Suri and Vijin Vincent. An Empirical Examination of the Relationship between Code Smells and Vulnerabilities. International Journal of Computer Applications 176(32):1-9, June 2020. DOI: 10.5120/ijca2020920362

[3] Android Developers. The contribution of android to economic growth. Available at androiddeveloper.galileo.edu/2017/05/12/the-contribution-of- android-to-economicgrowth/, 2017.

[4] Carette A, Younes MA, Hecht G, Moha N, Rouvoy R. Investigating the energy impact of android smells. In2017 IEEE 24th International Conference on Software Analysis, Evolution and Reengineering (SANER), 2017 (pp. 115-126). IEEE.

[5] Chowdhury I, Zulkernine M. Using complexity, coupling, and cohesion metrics as early indicators of vulnerabilities. Journal of Systems Architecture. 2011, 57(3):294313.

[6] Craig. Chapple. Global app revenue grew 23 billion. Available at sensortower.com/blog/app-revenue-and-downloads- q3-2019.

[7] Fontana FA, Mäntylä MV, Zanoni M, Marino A. Comparing and experimenting machine learning techniques for code smell detection. Empirical Software Engineering.; ,2016, 21(3):1143-91.

[8] Fontana FA, Walter B, Zanoni M. Code smells and micro patterns correlations. InRefTest 2013 Workshop, co-located event with XP 2013 Conference, 2013. 
[9] Fowler M. Refactoring. Improving the design of existing code. In1 1th European Conference. Jyväskylä, Finland, 1997.

[10] Gupta A, Suri B, Kumar V, Jain P. Extracting rules for vulnerabilities detection with static metrics using machine learning. International Journal of System Assurance Engineering and Management, 2020.

[11] Gupta A, Suri B, Kumar V, Misra S, Blažauskas T, Damaševičius R. Software code smell prediction model using Shannon, Rényi and Tsallis entropies. Entropy, 2018.20(5):372.

[12] Harer JA, Kim LY, Russell RL, Ozdemir O, Kosta LR, Rangamani A, Hamilton LH, Centeno GI, Key JR, Ellingwood PM, Antelman E. Automated software vulnerability detection with machine learning. arXiv preprint arXiv:1803.04497. 2018 Feb 14.

[13] Hei X, Du X, Lin S. Two vulnerabilities in Android OS kernel. In2013 IEEE International Conference on Communications, 2013(ICC (pp. 6123-6127). IEEE.

[14] Hornik K, Stinchcombe M, White H. Multilayer feedforward networks are universal approximators. Neural networks. 1989 Jul 1;2(5):359-66.

[15] Huang H, Zhu S, Chen K, Liu P. From system services freezing to system server shutdown in android: All you need is a loop in an app. InProceedings of the 22nd ACM SIGSAC Conference on Computer and Communications Security, 2015 (pp. 1236-1247).

[16] Islam MR, Zibran MF. A comparative study on vulnerabilities in categories of clones and non-cloned code. In 2016 IEEE 23rd International Conference on Software Analysis, Evolution, and Reengineering (SANER) 2016, (Vol. 3, pp. 8-14). IEEE.

[17] Jošt GR, Huber J, HeriČko M. Using object oriented software metrics for mobile application development. In 2nd Workshop of Software Quality Analysis, Monitoring, Improvement, and Applications, 2013 (pp. 17-27).

[18] Khashei M, Bijari M. An artificial neural network (p, d, q) model for timeseries forecasting. Expert Systems with applications, 2010 .37(1):479-89.

[19] Lee S, Choeh JY. Predicting the helpfulness of online reviews using multilayer perceptron neural networks. Expert Systems with Applications. 2014 ;41(6):3041-6.

[20] Li Z, Zou D, Xu S, Ou X, Jin H, Wang S, Deng Z, Zhong Y. Vuldeepecker: A deep learning-based system for vulnerability detection. arXiv preprint arXiv:1801.01681. 2018 Jan 5.

[21] Lieberherr KJ, Holland IM. Assuring good style for object-oriented programs. IEEE software. $1989 \mathrm{Sep} ; 6(5): 38-48$.

[22] Linares-Vásquez M, Bavota G, Escobar-Velásquez C.. An empirical study on androidrelated vulnerabilities. In2017 IEEE/ACM 14th International Conference on Mining Software Repositories (MSR) 2017. (pp. 2-13). IEEE.

[23] Mahmood R, Mahmoud QH. Evaluation of static analysis tools for finding vulnerabilities in Java and C/C++ source code. arXiv preprint arXiv:1805.09040. 2018 May 23. 
[24] Meshram PD, Thool RC. A survey paper on vulnerabilities in android OS and security of android devices. In2014 IEEE Global Conference on Wireless Computing \& Networking (GCWCN). 2014 (pp. 174-178). IEEE.

[25] Mohit Maheshwari. Top programming languages for android app development. Available at dzone.com/articles/most-used-programming-languages-for-android-appde.

[26] Palomba F, Bavota G, Di Penta M, Oliveto R, De Lucia A, Poshyvanyk D. Detecting bad smells in source code using change history information. In2013 28th IEEE/ACM International Conference on Automated Software Engineering (ASE) 2013.(pp. 268278). IEEE.

[27] Palomba F, Di Nucci D, Panichella A, Zaidman A, De Lucia A. Lightweight detection of android-specific code smells: The adoctor project. In2017 IEEE 24th international conference on software analysis, evolution and reengineering (SANER)2017. (pp. 487-491). IEEE.

[28] Palomba F, Oliveto R, De Lucia A. Investigating code smell co-occurrences using association rule learning: A replicated study. In 2017 IEEE Workshop on Machine Learning Techniques for Software Quality Evaluation (MaLTeSQuE) 2017 (pp. 8-13). IEEE.

[29] Pang Y, Xue X, Wang H. Predicting vulnerable software components through deep neural network. InProceedings of the 2017 International Conference on Deep Learning Technologies 2017 Jun 2 (pp. 6-10).

[30] Park H, Baek S.An empirical validation of a neural network model for software effort estimation. Expert Systems with Applications.2008, 35(3):929-37.

[31] PI LLC. (2014). The security impact of mobile device use by employ- ees. Ponemon Institute, Tech. Rep., 2014.

[32] Reimann J, Brylski M, Aßmann U. A tool-supported quality smell catalogue for android developers. InProc. of the conference Modellierung 2014 in the Workshop Modellbasierte und modellgetriebene Softwaremodernisierung-MMSM

[33] Rob Sobers. (2020). 110 must-know cybersecurity statistics for 2020. Available at www.varonis.com/blog/cybersecurity-statistics/.

[34] Robert JS. Pattern Recognition. Statistical, Structural and Neural Approaches. New York. 1992.

[35] Rutar N, Almazan CB, Foster JS. A comparison of bug finding tools for java. In15th International Symposium on Software Reliability Engineering, 2004 (pp. 245-256). IEEE.

[36] Saccente N, Dehlinger J, Deng L, Chakraborty S, Xiong Y. Project achilles: A prototype tool for static method-level vulnerability detection of java source code using a recurrent neural network. In2019 34th IEEE/ACM International Conference on Automated Software Engineering Workshop (ASEW) 2019 Nov 11 (pp. 114-121). IEEE. 
[37] Sahraoui HA, Godin R, Miceli T. Can metrics help bridging the gap between the improvement of $\mathrm{OO}$ design quality and its automation. InProceedings of the International Conference on Software Maintenance, ICSM, 2000

[38] Scheffer T. Finding association rules that trade support optimally against confidence. In European conference on principles of data mining and knowledge Discovery, 2001 (pp. 424-435). Springer, Berlin, Heidelberg.

[39] Shewale H, Patil S, Deshmukh V, Singh P. Analysis of android vulnerabilities and modern exploitation techniques. ICTACT journal on communication technology, 2014. 5(1):863-7.

[40] Skybox Security. vulnerability and threat trends. Available at lp.skyboxsecurity.com/rs/440-MPQ- 510/images/2020V TT rends Report reduced.pdf., 2020.

[41] Szőke G, Nagy C, Fülöp LJ, Ferenc R, Gyimóthy T. FaultBuster. An automatic code smell refactoring toolset. In 2015 IEEE 15th International Working Conference on Source Code Analysis and Manipulation (SCAM) 2015, (pp. 253-258). IEEE.

[42] Wang Q, Yu B, Zhu J. Extract rules from software quality prediction model based on neural network. In16th IEEE International Conference on Tools with Artificial Intelligence .2004 (pp. 191-195). IEEE.

[43] Wu, F., Wang, J., Liu, J. and Wang, W., December. Vulnerability detection with deep learning. In 2017 3rd IEEE International Conference on Computer and Communications (ICCC), 2017. (pp. 1298-1302).

[44] Yamashita A, Moonen L. Do developers care about code smells? An exploratory survey. In2013 20th Working Conference on Reverse Engineering (WCRE) , 2013(pp. 242-251). IEEE.

Received 15.01.2021, Accepted 30.09.2021 